\title{
Logic without metaphysics
}

\author{
José L. Zalabardo ${ }^{1}$
}

Received: 31 August 2018 / Accepted: 2 February 2019 / Published online: 9 February 2019

(c) The Author(s) 2019

\begin{abstract}
Standard definitions of logical consequence for formal languages are atomistic. They take as their starting point a range of possible assignments of semantic values to the extralogical atomic constituents of the language, each of which generates a unique truth value for each sentence. In modal logic, these possible assignments of semantic values are generated by Kripke-style models involving possible worlds and an accessibility relation. In first-order logic, they involve the standard structures of model theory, as sets of objects from which the extralogical symbols of the language receive their denotations. I argue that there is an alternative, holistic, approach to the task of defining logical consequence for a formal language. It specifies necessary and sufficient conditions for an assignment of truth values to all the sentences of the language to be compatible with the intended interpretation of its logical constants. It achieves this without invoking possible assignments of semantic values to the extralogical atomic constituents of the language, or the formal resources that are employed to generate these. I show how this approach can be successfully applied to modal propositional logic and to first-order logic, modal as well as nonmodal. I show that the holistic definitions of logical consequence that I supply for these languages are equivalent to the standard atomistic definitions.
\end{abstract}

Keywords Modality · Necessity · Logical consequence · Formal semantics · Substitutional quantification · Possible worlds $\cdot$ Modal semantics

\section{Atomism and holism in formal semantics}

The subject matter of this paper is the contrast between two approaches to the task of defining the relation of logical consequence in a formal language. I am going to use for these approaches the labels atomistic and holistic. Logical consequence in a formal language is a binary relation pairing sets of sentences of the language (the premises)

José L. Zalabardo

j.zalabardo@ucl.ac.uk

1 Philosophy Department, UCL, Gower Street, London WC1E 6BT, UK 
with sentences of the language (the conclusion). It is intended to model the relation that obtains between a set of premises and a conclusion when the forms of premises and conclusion make it impossible for the conclusion to be false if the premises are all true. The atomistic approach and the holistic approach are two alternative strategies for the task of defining this relation.

Standard semantic definitions of logical consequence for formal languages follow the atomistic approach. ${ }^{1}$ Their starting point is a range of possible assignments of semantic values to the extralogical atomic constituents of the sentences of the language. I am going to refer to the items that play this role in the atomistic approach as ASAs (Atomic Semantic Assignments). In propositional logic, the role of ASAs is played by atomic valuations-functions from the set of atoms to the set $\{T, F\}$. In modal propositional logic, ASAs are possible worlds in Kripke-style modal models. In firstorder logic, ASAs are the usual structures of model theory, consisting of a set of objects and an assignment of extensions over this set to the extralogical symbols of the language. In modal first-order logic, ASAs are items that combine the features of modal propositional ASAs and nonmodal first-order ASAs.

In each case, ASAs are so chosen that for every ASA and every sentence of the language, there will be a unique truth value for the sentence that's compatible with the ASA and with the intended interpretations of the logical constants that figure in the sentence. The next step in the implementation of the atomistic approach is to specify, for each ASA and each sentence of the language, the truth value that the sentence will receive from the ASA. This is usually presented as a definition of truth for the language. Once this has been achieved, we are in a position to define logical consequence, saying that a sentence $\phi$ of the language is a logical consequence of a set $\Gamma$ of sentences of the language just in case every ASA is such that if it gives the value True to every element of $\Gamma$, then it also gives the value True to $\phi$.

The atomistic approach is almost universally employed. But is it compulsory? The main thesis of this paper is that it isn't: for propositional and first-order logic, modal as well as nonmodal, there is a viable alternative-the holistic approach. In the holistic approach, ASAs don't play any role. Its starting point is the set of valuations for the language in question - functions from the set of sentences of the language (all sentences, molecular as well as atomic) to the set $\{T, F\}$. Some valuations will be compatible with the intended interpretations of the logical constants of the language, while other valuations will be incompatible with these interpretations. This contrast is the central concept of the holistic approach. It proceeds by specifying necessary and sufficient conditions for a valuation to be compatible with the intended interpretations of the logical constants of the language. I shall refer to valuations that satisfy this condition as admissible. The holistic approach seeks to specify which valuations are admissible without invoking ASAs-by looking directly at how the intended interpretations of

\footnotetext{
1 Semantic definitions are those that take as their starting point the thought that $\phi$ is a logical consequence of $\Gamma$ just in case structural features of $\phi$ and of the elements of $\Gamma$ are incompatible with assigning to $\phi$ the value False if all the elements of $\Gamma$ receive the value True. In the present paper I'm restricting my attention to semantic definitions. The model-theoretic approach to logical consequence produces atomistic semantic definitions. The holistic definitions that I'm going to provide are also semantic. Proof-theoretic approaches produce non-semantic definitions of logical consequence, and fall outside the scope of this paper. It could be argued, although I won't do it here, that the holistic semantic approach that I'm going to present has some of the advantages claimed for the proof-theoretic approach.
} 
the logical constants rule out some combinations of truth values for the sentences of the language. Once we have specified which valuations are admissible, logical consequence can be defined directly, saying that a sentence $\phi$ is a logical consequence of a set of sentences $\Gamma$ just in case every admissible valuation is such that if all the elements of $\Gamma$ receive the value True from it, then $\phi$ also receives the value True.

My main goal in this paper is to show how the holistic approach can be successfully applied to the task of defining logical consequence for propositional and first-order languages, modal as well as nonmodal. In Sect. 2 I shall introduce the contrast between the two approaches in the context of (nonmodal) propositional logic, where they are virtually interchangeable. In Sect. 3, I provide a holistic definition of logical consequence for modal propositional logic. In Sect. 4, I apply the holistic approach to first-order logic. And in Sect. 5, I present a holistic treatment of modal first-order logic, combining the ideas deployed for modal propositional logic and nonmodal first-order logic. In each case I will start by providing a detailed presentation of the atomistic treatment of the language in question. These will be familiar to the reader, but they are offered for comparison and to facilitate the proofs of the equivalence of the holistic definitions I provide with the standard atomistic alternatives. Proving these results is the business of Sect. 7. Section 6 provides a brief discussion of the philosophical relevance of these formal results.

\section{Propositional logic}

In nonmodal propositional logic, the contrast between the atomistic approach and the holistic approach is of no great consequence and can easily go unnoticed. Nevertheless, both approaches have a clear application to this case. My goal in this section is to present how both the atomistic approach and the holistic approach can be used to define logical consequence in nonmodal propositional logic.

Let the language of propositional logic be the set $P L$ of sentences defined inductively in the usual way, with a denumerable set of atoms as the base and inductive clauses for the sentential connectives, say $\neg$ and $\wedge$.

\subsection{The atomistic approach}

Let's consider first how to apply the atomistic approach to the task of defining logical consequence for $P L$-sentences. In this case, the role of ASAs is played by atomic valuations - functions from the set of atoms to the set $\{T, F\}$. Having identified atomic valuations as ASAs, our next goal is to specify, for each atomic valuation and $P L$ sentence, the truth value that the sentence receives from the atomic valuation. To achieve this we define, for every atomic valuation $a$, the $P L$-valuation $v_{a}$ pairing each $P L$-sentence with the truth value that it will receive from $a$, given the intended interpretations of the logical constants that figure in it. We define $v_{a}$ by recursion, as the unique $P L$-valuation satisfying the following conditions:

(Atoms) For every atom $\alpha, v_{a}(\alpha)=a(\alpha)$. 
$(\neg)$ For every $P L$-sentence of the form $\neg \phi$,

$$
v_{a}(\neg \phi)= \begin{cases}T & \text { if } v_{a}(\phi)=F ; \\ F & \text { otherwise. }\end{cases}
$$

$(\wedge)$ For every $P L$-sentence of the form $\phi \wedge \psi$,

$$
v_{a}(\phi \wedge \psi)= \begin{cases}T & \text { if } v_{a}(\phi)=v_{a}(\psi)=T \\ F & \text { otherwise. }\end{cases}
$$

This definition specifies the truth conditions of any given $P L$-sentence $\phi$, as the set of atomic valuations from which $\phi$ receives the value $T$.

We can now use this notion to define logical consequence in the following way:

A $P L$-sentence $\phi$ is a logical consequence of a set of $P L$-sentences $\Gamma(\Gamma \vDash \phi)$ just in case for every atomic valuation $a$, if $v_{a}(\gamma)=T$ for every $\gamma \in \Gamma$, then $v_{a}(\phi)=T$.

\subsection{The holistic approach}

Let's consider now how the holistic approach could be used to define logical consequence in $P L$. On this approach, atomic valuations don't play any role. We consider all the possible $P L$-valuations and proceed by specifying necessary and sufficient conditions for a $P L$-valuation to count as admissible - to be compatible with the intended interpretations of the logical constants of the language. We achieve this with the concept of a Boolean $P L$-valuation, where a $P L$-valuation is Boolean just in case it satisfies the following conditions:

$(\neg)$ For every $P L$-sentence $\phi, v(\phi) \neq v(\neg \phi)$.

$(\wedge)$ For all $P L$-sentences $\phi, \psi, v(\phi \wedge \psi)=T$ iff $v(\phi)=v(\psi)=T$.

In spite of the obvious correlation between the definition of Boolean $P L$-valuation and the connective-clauses of the definition of $v_{a}$, it is important to keep in mind the difference between the roles that they play. The connective-clauses of the definition of $v_{a}$ are part of a recursive definition of a function from $P L$ to $\{T, F\}$ (one function for each atomic valuation). For each inductive clause of the definition of $P L$, they specify the image of the output of the clause as a function of the image of the input of the clause. The clauses of the definition of Boolean $P L$-valuation play a different role. They simply rule out $P L$-valuations in which certain combinations of values are present.

If we take the admissible $P L$-valuations to be the Boolean ones, we can now provide a holistic definition of logical consequence for $P L$ :

A $P L$-sentence $\phi$ is a logical consequence of a set of $P L$-sentences $\Gamma(\Gamma \vDash \phi)$ just in case for every Boolean valuation $v$, if $v(\gamma)=T$ for every $\gamma \in \Gamma$, then $v(\phi)=T$. 
The atomistic and holistic definitions of logical consequence for $P L$ are clearly equivalent. This follows directly from the following trivial result:

Proposition 1 A PL-valuation $v$ is Boolean just in case for some atomic valuation a, $v=v_{a}$.

Generally, there isn't much to choose between the two definitions. By bypassing ASAs, the holistic approach has the potential for greater parsimony. However, this potential is not realised in nonmodal propositional logic, since invoking atomic valuations doesn't seem to bring about a substantial increase in the complexity of the resulting definition of logical consequence. We shall see in the remainder that for other formal languages the situation is very different in this regard.

\section{Modal propositional logic}

Modal propositional logic extends propositional logic with a sentential connective whose intended interpretation models the behaviour of the expression necessarily.

The language of modal propositional logic is the set $M P L$ of sentences defined inductively in the same way as $P L$, with an additional inductive clause for the necessity operator, $\square$. An $M P L$-valuation is a function from $M P L$ to $\{T, F\}$.

Our goal in the present section is to present the contrast between the atomistic approach and the holistic approach as it applies to modal propositional logic. Here the atomistic approach reigns supreme. Logical consequence for modal propositional languages is only ever defined along the lines of the atomistic approach. Hence it might come as a surprise that the holistic approach is also applicable in this case. My main goal will be to present a holistic definition of logical consequence for $M P L$ which will be shown in Sect. 7 to be equivalent to the standard atomistic definition.

\subsection{The atomistic approach}

In order to apply the atomistic approach to $M P L$, our first task is to identify the items that we are going to use as ASAs in this case. Here atomic valuations won't do. The necessity operator is not truth-functional. Therefore there isn't a unique $M P L$-valuation extending a given atomic valuation that is compatible with the intended interpretations of the logical constants of $M P L$.

The standard strategy for overcoming this obstacle, due to Kripke (1963), is to invoke at this point the notion of a (modal) model. A (modal) model is a triple $M=$ $\left\langle W_{M}, R_{M}, A_{M}\right\rangle$, where $W_{M}$ is a nonempty set (the possible worlds), $R_{M}$ is a binary relation on $W_{M}$ (accessibility) and $A_{M}$ is a function pairing each atom and possible world with a unique truth value.

An ASA, on this implementation of the atomistic approach, is a model and a world in this model. Each world in each model will assign a unique truth value to each $M P L$-sentence, and the next item of business for the atomistic approach is to define, for each model, the function that pairs each world and $M P L$-sentence with the value that the sentence receives at the world. 
If $M$ is the model $\left\langle W_{M}, R_{M}, A_{M}\right\rangle$, the modal valuation function for $M$ is the function $V_{M}$ pairing each $M P L$-sentence and element of $W_{M}$ with a truth value that satisfies the following conditions:

(Atoms) For every atom $\alpha$ and $w \in W_{M}, V_{M}(\alpha, w)=A_{M}(\alpha, w)$.

The truth-functional connectives are handled in the same way as in $P L$. For $\square$ we have:

( $\square$ ) For every $M P L$-sentence $\phi$ and $w \in W_{M}$,

$$
V_{M}(\square \phi, w)= \begin{cases}T & \text { if for every } w^{\prime} \in W_{M} \text { such that } w R_{M} w^{\prime}, V_{M}\left(\phi, w^{\prime}\right)=T ; \\ F & \text { otherwise. }\end{cases}
$$

Using the notion of the modal valuation function for a model we can provide an atomistic definition of logical consequence for $M P L$ :

An $M P L$-sentence $\phi$ is a $K$-logical consequence of a set of $M P L$-sentences $\Gamma$ $\left(\Gamma \vDash_{K} \phi\right)$ just in case for every model $M$ and every $w \in W_{M}$, if $V_{M}(\gamma, w)=T$ for every $\gamma \in \Gamma$, then $V_{M}(\phi, w)=T$.

This definition corresponds to the weakest normal modal system $K$. Definitions corresponding to stronger modal systems can be easily provided by imposing conditions on the accessibility relation of the models in terms of which logical consequence is defined.

\subsection{The holistic approach}

In a strict application of the holistic template to this case, our starting point would have to be a specification of necessary and sufficient conditions for an $M P L$-valuation to be compatible with the intended interpretations of the logical constants of $M P L$. But this approach faces a serious obstacle. The problem is that we want the following principle to hold for the relation of logical consequence that we are seeking to define:

(K) For every $M P L$-sentence $\phi$ and every set $\Gamma$ of $M P L$-sentences, if $\Gamma \vDash \phi$, then $\{\square \gamma: \gamma \in \Gamma\} \vDash \square \phi$.

A condition on admissible $M P L$-valuations that secured this result would have to rule out an MPL-valuation $v$ such that $v(\square \gamma)=T$ for every $\gamma \in \Gamma$ and $v(\square \phi)=F$ if $\Gamma \vDash \phi$. But the condition cannot be formulated in this way on pain of circularity, since we are hoping to define $\vDash$ in terms of the notion of an admissible $M P L$-valuation. A direct application of the holistic approach to $M P L$ would require finding a formulation of the relevant constraint that doesn't exhibit this kind of circularity.

Here I'm going to present a different strategy for overcoming the obstacle. ${ }^{2} \mathrm{~A}$ minor adjustment to the holistic template will suffice to obtain the intended result. The proposal is to impose necessary and sufficient conditions, not directly on individual $M P L$-valuations, but on sets of $M P L$-valuations. Then we'll be able to say that an $M P L$-valuation is compatible with the intended interpretations of the logical constants of $M P L$ just in case it is an element of a set that satisfies these conditions.

\footnotetext{
${ }^{2}$ I sketch this strategy in Zalabardo (2018).
} 
The sets we are after will have to contain nothing but Boolean $M P L$-valuations. Otherwise the valuations in the set won't be compatible with the intended interpretations of the truth-functional connectives. In order to ensure that the valuations are also compatible with the intended interpretation of the necessity operator we need to impose a condition on the set as a whole. The crucial concept for formulating this condition is the relation of actualization between $M P L$-valuations:

An $M P L$-valuation $v^{\prime}$ actualizes an $M P L$-valuation $v$ just in case, for every $M P L$ sentence $\phi$, if $v(\square \phi)=T$ then $v^{\prime}(\phi)=T$.

Thus $v^{\prime}$ actualizes $v$ just in case all the necessities of $v$ are true in $v^{\prime}$. Using the notion of actualization we can define necessary and sufficient conditions on a set of $M P L$-valuations that will ensure that an $M P L$-valuation will be compatible with the intended interpretations of the logical constants of $M P L$ just in case it is an element of one of these sets. I shall refer to sets of $M P L$-valuations satisfying these conditions as $m$-Boolean.

A nonempty set $V$ of Boolean $M P L$-valuations is $m$-Boolean just in case it satisfies the following condition:

For every MPL-sentence $\phi$ and every $v \in V$, if for every $v^{\prime} \in V$ such that $v^{\prime}$ actualizes $v, v^{\prime}(\phi)=T$, then $v(\square \phi)=T$.

Notice that we don't need to require, in addition, that $\square \phi$ is false in $v$ if $\phi$ is false in some valuation that actualizes $v$. If $\square \phi$ is true in $v$ and $\phi$ is false in $v^{\prime}$, then $v^{\prime}$ doesn't actualize $v$. This means that a set of Boolean valuations can fail to be m-Boolean only if it contains a valuation that fails to treat as necessary what's true in every valuation that actualizes it.

I'd like to emphasize that an m-Boolean set does not consist in a set of Boolean $M P L$-valuations and a binary relation on this set (actualization) that satisfy certain conditions. ${ }^{3}$ We are not making covert appeal to the relation of accessibility used by the atomistic approach. An m-Boolean set is simply a set of Boolean MPL-valuations that satisfies a certain condition. We have introduced the notion of actualization in order to provide a perspicuous formulation of the condition that a set of Boolean $M P L$-valuations needs to satisfy in order to be m-Boolean. But the notion is entirely dispensable. The condition that we've used to define m-Boolean sets can be easily formulated without its help ( $\phi$ and $\psi$ range over $M P L$-sentences and $v$ and $v^{\prime}$ over elements of $V)$ :

$$
\forall \phi \forall v\left(\forall v^{\prime}\left[\left(\forall \psi\left(v(\square \psi)=T \rightarrow v^{\prime}(\psi)=T\right) \rightarrow v^{\prime}(\phi)=T\right] \rightarrow v(\square \phi)=T\right)\right.
$$

Using the notion of m-Boolean sets of $M P L$-valuations we can provide a holistic definition of logical consequence for $M P L$ corresponding to the weakest normal system $K$ :

An $M P L$-sentence $\phi$ is a $K$-logical consequence of a set of $M P L$-sentences $\Gamma$ $\left(\Gamma \vDash_{K} \phi\right.$ ) just in case for every m-Boolean set of $M P L$-valuations $V$ and every $v \in V$, if $v(\gamma)=T$ for every $\gamma \in \Gamma$, then $v(\phi)=T$.

\footnotetext{
${ }^{3}$ My proposal differs in this respect from Hughes Leblanc's truth-functional semantics for modal logic (Leblanc 1976), which invokes a set of (atomic) valuations and a binary relation on this set.
} 
And we can produce definitions corresponding to stronger modal systems in terms of suitably restricted classes of m-Boolean sets, by imposing the same conditions on actualization that are used with accessibility in the atomistic approach.

Here, however, a different strategy is also possible. For $T$, for example, we can use sets of $M P L$-valuations which, in addition to being Boolean, satisfy the following condition:

(T) For every $M P L$-sentence $\phi$, if $v(\square \phi)=T$ then $v(\phi)=T$.

Likewise for $\mathrm{S} 4$ we can focus on sets of Boolean valuations that satisfy $(T)$ and:

(4) For every $M P L$-sentence $\phi$, if $v(\square \phi)=T$ then $v(\square \square \phi)=T$.

Other normal systems can be treated in the same way.

The holistic definition of K-logical consequence presented in this section is equivalent to the atomstic definition. This is expressed by the following result:

Theorem 1 For every MPL-sentence $\phi$ and every set of MPL-sentences $\Gamma$, the following are equivalent:

1. For every modal model $M$ and every $w \in W_{M}$, if $V_{M}(\gamma, w)=T$ for every $\gamma \in \Gamma$, then $V_{M}(\phi, w)=T$.

2. For every $m$-Boolean set $V$ of $M P L$-valuations and every $v \in V$, if $v(\gamma)=T$ for every $\gamma \in \Gamma$, then $v(\phi)=T$.

In Sect. 7.1, below, I offer a proof of this result.

\section{First-order logic}

In this section I present the contrast between the atomistic and the holistic approach for (nonmodal) first-order logic. We consider first-order languages whose logical vocabulary consists of the connectives and brackets of $P L$, the universal quantifier $\forall$, the identity sign, $\doteq$, and denumerably many variables. The extralogical vocabulary of a first-order language may contain $n$-place predicates, for any positive integer $n$, and individual constants. For any extralogical vocabulary the terms of the language are the variables and the individual constants of the vocabulary. The set of formulas of the language is defined by induction in the usual way. Then the set of sentences of the language is defined as the set of formulas with no free variables.

Our goal in this section is to present the application of the atomistic approach and the holistic approach to the task of defining logical consequence as a relation between sets of sentences of a first-order language $L$ and sentences of $L$. The holistic definition of logical consequence presented in this section will be shown in Sect. 7.2 to be equivalent to the standard atomistic definition.

\subsection{The atomistic approach}

In the standard application of the atomistic approach to the task of defining logical consequence for a first-order language $L$, the role of ASAs is played by $L$-structures. 
An $L$-structure $\mathfrak{A}$ consists in a non-empty set $A$, known as the universe of $\mathfrak{A}$, and an interpretation in $A$ for every extralogical symbol $s$ of $L, s_{\mathfrak{A}}$, as follows:

- For every individual constant $c$ of $L, c_{\mathfrak{A}} \in A$.

- For every $n$-place predicate $P$ of $L, P_{\mathfrak{A}} \in \mathscr{P}\left(A^{n}\right)$.

The next step in the implementation of the atomistic approach is to specify, for each $L$-structure and each $L$-sentence, the truth value that the sentence receives from the structure. This has to be done indirectly, defining in the first instance the value that each $L$-formula receives from an $L$-structure relative to an interpretation of the variables in the universe of the structure. If $\mathfrak{A}$ is an $L$-structure, a variable-interpretation in $\mathfrak{A}$ is a function from the set of variables to the universe of $\mathfrak{A}$.

The denotation in $\mathfrak{A}$ of an $L$-term $t$ relative to a variable-interpretation $s$ in $\mathfrak{A}$, $\operatorname{den}_{\mathfrak{A}}(t, s)$, can be defined as follows:

(IC) For every individual constant $c$ of $L, \operatorname{den}_{\mathfrak{A}}(c, s)=c_{\mathfrak{A}}$.

(Var) For every variable $x, \operatorname{den}_{\mathfrak{A}}(x, s)=s(x)$.

We now define by recursion the truth value in $\mathfrak{A}$ of an $L$-formula $\phi$ relative to a variable-interpretation $s$ in $\mathfrak{A}, v_{\mathfrak{A}}(\phi, s)$. If $s$ is a variable-interpretation in $\mathfrak{A}$, then, for all variables $x, y$ and every $a \in A$,

$$
s_{(x / a)}(y)= \begin{cases}a & \text { if } x=y \\ s(y) & \text { otherwise }\end{cases}
$$

$v_{\mathfrak{A}}(\phi, s)$ is the unique function satisfying the following conditions:

(Pred) For every $L$-formula of the form $P t_{1} \ldots t_{n}$,

$$
v_{\mathfrak{A}}\left(P t_{1} \ldots t_{n}, s\right)= \begin{cases}T & \text { if }\left\langle\operatorname{den}_{\mathfrak{A}}\left(t_{1}, s\right), \ldots, \operatorname{den}_{\mathfrak{A}}\left(t_{n}, s\right)\right\rangle \in P_{\mathfrak{A}} \\ F & \text { otherwise }\end{cases}
$$

$(\doteq)$ For every $L$-formula of the form $t \doteq u$,

$$
v_{\mathfrak{A}}(t \doteq u, s)= \begin{cases}T & \text { if } \operatorname{den}_{\mathfrak{A}}(t, s)=\operatorname{den}_{\mathfrak{A}}(u, s) \\ F & \text { otherwise }\end{cases}
$$

The sentential connectives are handled in the same way as in $P L$. For the universal quantifier we have:

( $\forall$ ) For every $L$-formula of the form $\forall x \phi$,

$$
v_{\mathfrak{A}}(\forall x \phi, s)= \begin{cases}T & \text { if for every } a \in A, v_{\mathfrak{A}}\left(\phi, s_{(x / a)}\right)=T ; \\ F & \text { otherwise. }\end{cases}
$$

Now, in general, the value that $s$ gives to variables that are free in an $L$-formula $\phi$ could affect the value of $v_{\mathfrak{A}}(\phi, s)$. Hence it's not the case for every $L$-formula $\phi$ and all 
variable-interpretations $s, s^{\prime}$ in $\mathfrak{A}$ that $v_{\mathfrak{A}}(\phi, s)=v_{\mathfrak{A}}\left(\phi, s^{\prime}\right)$. However for $L$-sentences, $L$-formulas with no free variables, this does hold in general: For every $L$-sentence $\phi$ and all variable-interpretations $s, s^{\prime}$ in $\mathfrak{A}$, we have that $v_{\mathfrak{A}}(\phi, s)=v_{\mathfrak{A}}\left(\phi, s^{\prime}\right)$. This means that in defining the binary function $v_{\mathfrak{A}}$ pairing $L$-formulas and variable-interpretations with truth values, we have defined a unary function, call it $v s_{\mathfrak{A}}$, pairing each $L$-sentence with a unique truth value, as required by the atomistic approach. $v s_{\mathfrak{A}}$ gives the truth conditions of each $L$-sentence $\phi$, by specifying the truth value that $\phi$ receives from each $L$-structure.

Now we can define the relation of logical consequence for sentences of a first-order language $L$ as follows:

An $L$-sentence $\phi$ is a logical consequence of a set of $L$-sentences $\Gamma(\Gamma \vDash \phi)$ just in case for every $L$-structure $\mathfrak{A}$, if $v s_{\mathfrak{A}}(\gamma)=T$ for every $\gamma \in \Gamma$, then $v s_{\mathfrak{A}}(\phi)=T .{ }^{4}$

\subsection{The holistic approach}

In the holistic approach ASAs play no role. Hence in the case of first-order logic, $L$-structures have no role to play in a holistic definition of logical consequence for a first-order language $L$. The starting point of the holistic approach is the set of $L$ valuations-functions from the set of $L$-sentences to the set $\{T, F\}$. The holistic approach proceeds by specifying necessary and sufficient conditions for an $L$-valuation to count as admissible - as compatible with the intended interpretations of the logical symbols of a first-order language. To achieve this, we need the concept of substitution:

For every $L$-formula $\phi$, individual constant $c$ of $L$ and variable $x$, the $c / x$-substitution of $\phi,(\phi)[c / x]$, is the formula that we obtain by replacing $x$ with $c$ wherever it occurs in free $\phi$.

We are now in a position to specify necessary and sufficient conditions for an $L$ valuation to count as admissible - as compatible with the intended interpretations of the logical constants of a first-order language. Our proposal will fall somewhat short of this goal, but we will then be able to overcome this shortcoming with our definition of logical consequence.

We are going to use for this purpose the notion of a $q$-Boolean $L$-valuation, defined, as with Boolean $P L$-valuations, with a list of individually necessary and jointly sufficient conditions, specifying which $L$-valuations are ruled out by the intended interpretation of each logical constant. For the connectives we use the same conditions as in propositional logic. We just need to add to these one for the universal quantifier and two for the identity sign.

An $L$-valuation $v$ is $q$-Boolean just in case it satisfies the following conditions:

$(\neg)$ For every $L$-sentence $\phi, v(\phi) \neq v(\neg \phi)$.

$(\wedge)$ For all $L$-sentences $\phi, \psi, v(\phi \wedge \psi)=T$ iff $v(\phi)=v(\psi)=T$.

$(\forall)$ For every $L$-formula $\phi$ in which no variable other than $x$ is free, $v(\forall x \phi)=T$ iff for every individual constant $c$ of $L, v((\phi)[c / x])=T$.

\footnotetext{
4 In standard applications of the atomistic approach, logical consequence is defined for all $L$-formulas. Here I've restricted the definition to $L$-sentences in order to facilitate comparisons with the holistic approach.
} 
$(\doteq a)$ For every individual constant $c$ of $L, v(c \doteq c)=T$.

$(\doteq b)$ For all individual constants $c, c^{\prime}$ of $L$, if $v\left(c \doteq c^{\prime}\right)=T$, then for every $L$-formula $\phi$ in which no variable other than $x$ is free, $v((\phi)[c / x])=$ $v\left((\phi)\left[c^{\prime} / x\right]\right)$.

Clause $(\forall)$ clearly takes the approach of the substitutional interpretation of the quantifiers. Notice, though, that interpreting the quantifiers substitutionally doesn't by itself result in a version of the holistic approach. Substitutional quantification is standardly presented in the context of an atomistic semantics for first-order logic, which differs from the standard objectual approach only in treating as ASAs, not first-order structures, but atomic valuations. ${ }^{5}$

Treating q-Boolean $L$-valuations as admissible, a straightforward application of the holistic template would produce the following definition of logical consequence:

An $L$-sentence $\phi$ is an $L$-logical consequence of a set $\Gamma$ of $L$-sentences $\left(\Gamma \models^{L} \phi\right)$ just in case for every q-Boolean $L$-valuation $v$, if $v(\gamma)=T$ for every $\gamma \in \Gamma$, then $v(\phi)=T$.

However, $L$-logical consequence doesn't quite grasp the intuitive notion of logical consequence. To see this, notice that every instance of the following schema, where $\phi$ is an $L$-formula in which only $x$ is free, is a true $L$-logical consequence claim:

$$
\{(\phi)[c / x]: c \text { is an individual constant of } L\} \vDash^{L} \forall x \phi
$$

The definition of q-Boolean $L$-valuation entails directly that every q-Boolean $L$ valuation making all the premises in an instance of this schema true will also make the conclusion true. But intuitively this set of premises should not logically entail this conclusion. The universal conclusion can have a counterexample in an object for which the language doesn't have a name. Then the conclusion should be false even if all the premises are true. The premises say that all the objects for which the language has names satisfy the condition expressed by $\phi .{ }^{6}$ But on the intuitive understanding of the universal quantifier the conclusion says more-that every object, whether or not the language has a name for it, satisfies the condition expressed by $\phi$. Logical relations should not be affected by which objects the language is capable of naming.

This has been traditionally a major objection to the substitutional interpretation of the quantifiers. However, the problem has a very simple solution, outlined by Dunn and Belnap (1968, p. 183). ${ }^{7}$ Let's say that first-order language $L^{\prime}$ is an onomastic expansion of first-order language $L$ just in case their vocabularies are identical except that the vocabulary of $L^{\prime}$ contains individual constants that are not in the vocabulary of $L$. Using this concept we can formulate a definition of logical consequence for $L$ that overcomes the obstacle we've encountered:

\footnotetext{
5 Dunn and Belnap's (1968) presentation of substitutional quantification follows this atomistic template (p. 179). The same goes for Leblanc (1976). Leblanc (1983, pp. 213-214) briefly mentions a holistic version of substitutional semantics that is essentially the one developed here. Holistic ideas can also be found in the work of Smullyan (1968, p. 47) and Hintikka (1955).

6 In the limiting case, for first-order languages with no individual constants, a q-Boolean valuation will give the value $T$ to every universal sentence.

7 Hughes Leblanc has provided a detailed development of this idea (see Leblanc 1983). Bonevac (1984) uses onomastic expansions in the quantifier-clauses of the definition of admissible valuations.
} 
An $L$-sentence $\phi$ is a logical consequence of a set $\Gamma$ of $L$-sentences $(\Gamma \vDash \phi)$ just in case for every onomastic expansion $L^{\prime}$ of $L, \Gamma \vDash^{L^{\prime}} \phi$.

The claim that this holistic definition of first-order logical consequence is equivalent to the atomistic definition provided above can be expressed as follows:

Theorem 2 For every sentence $\phi$ of a first-order language $L$ and every set $\Gamma$ of $L$ sentences the following are equivalent:

1. For every onomastic expansion $L^{\prime}$ of $L$, for every $q$-Boolean $L^{\prime}$-valuation $v$, if $v(\gamma)=T$ for every $\gamma \in \Gamma$, then $v(\phi)=T$.

2. For every $L$-structure $\mathfrak{A}$, if $v s_{\mathfrak{A}}(\gamma)=T$ for every $\gamma \in \Gamma$, then $v s_{\mathfrak{A}}(\phi)=T$.

In Sect. 7.2, below, I provide a proof of this result.

\section{Modal first-order logic}

We consider now modal first-order languages whose logical vocabulary contains the connectives and brackets of $P L$, the necessity operator $\square$, the universal quantifier $\forall$, the identity sign $\doteq$, and denumerably many variables. The extralogical vocabulary of a modal first-order language may contain $n$-place predicates, for any positive integer $n$, and individual constants.

As in nonmodal first-order languages, the terms of a modal first-order language are the variables and the individual constants. The set of formulas is also defined with the usual induction, including this time inductive clauses for both $\forall$ and $\square$. As with nonmodal first-order logic, the sentences of a modal first-order language are the formulas with no free variables.

\subsection{The atomistic approach}

Standard definitions of logical consequence for modal first-order languages follow the atomistic approach. The role of ASAs is played by items that combine the ideas of first-order structures and modal models. There are several important decisions one needs to make in order to effect this combination. Here we are going to present a particularly simple version of the idea. ${ }^{8}$

If $L$ is a modal first-order language, an $L$-model $M$ is a quadruple

$$
\left\langle W_{M}, D_{M}, R_{M}, I_{M}\right\rangle
$$

where:

- $W_{M}$ is a nonempty set (the possible worlds).

- $D_{M}$ is a nonempty set (the universe).

- $R_{M}$ is a binary relation on $W_{M}$ (accessibility).

$-I_{M}$ is an interpretation of each extralogical symbol of $L$, as follows:

\footnotetext{
8 On the approach discussed here, see Linsky and Zalta (1994). For other options see Garson (1984).
} 
- For every individual constant $c$ of $L, c_{M} \in D_{M}$. I.e. $c$ is interpreted with an object in the universe.

- For every $n$-place predicate $P$ of $L, P_{M} \in W_{M} \mathscr{P}\left(D_{M}^{n}\right)$. I.e. $P$ is interpreted with a function pairing each element of $W_{M}$ with a set of $n$-tuples of elements of $D_{M}$-its extension at that world. We shall refer to the set of $n$-tuples of elements of $D_{M}$ that $P_{M}$ pairs with $w$ as $P_{M}^{w}$.

A variable-interpretation in $M$ is a function from the set of variables to $D_{M}$.

The denotation in $M$ of an $L$-term $t$ at a world $w$ relative to a variable-interpretation $s$ in $M, \operatorname{den}_{M}(t, w, s)$, is defined as follows:

(IC) For every individual constant $c$ of $L, \operatorname{den}_{M}(c, w, s)=c_{M}$.

(Var) For every variable $x, \operatorname{den}_{M}(x, w, s)=s(x)$.

We now define by recursion the truth value in $M$ of an $L$-formula $\phi$ at a world $w$ relative to a variable-interpretation $s$ in $M, V_{M}(\phi, w, s)$, as the unique function satisfying the following conditions:

(Pred) For every $L$-formula of the form $P t_{1} \ldots t_{n}$,

$$
V_{M}\left(P t_{1} \ldots t_{n}, w, s\right)= \begin{cases}T & \text { if }\left\langle\operatorname{den}_{M}\left(t_{1}, w, s\right), \ldots, \operatorname{den}_{M}\left(t_{n}, w, s\right)\right\rangle \in P_{M}^{w} \\ F & \text { otherwise }\end{cases}
$$

$(\doteq)$ For every $L$-formula of the form $t \doteq u$,

$$
V_{M}(t \doteq u, w, s)= \begin{cases}T & \text { if } \operatorname{den}_{M}(t, w, s)=\operatorname{den}_{M}(u, w, s) \\ F & \text { otherwise. }\end{cases}
$$

The truth-functional connectives are handled in the same way as previously. For the universal quantifier and the necessity operator we have:

( $\forall$ ) For every $L$-formula of the form $\forall x \phi$,

$$
V_{M}(\forall x \phi, w, s)= \begin{cases}T & \text { if for every } a \in D_{M}, V_{M}\left(\phi, w, s_{(x / a)}\right)=T \\ F & \text { otherwise. }\end{cases}
$$

( $\square$ ) For every $L$-formula of the form $\square \phi$,

$$
V_{M}(\square \phi, w, s)= \begin{cases}T & \text { if for every } w^{\prime} \in W_{M} \text { such that } w R_{M} w^{\prime}, \\ & V_{M}\left(\phi, w^{\prime}, s\right)=T ; \\ F & \text { otherwise. }\end{cases}
$$

As in nonmodal first-order logic, variable-interpretations won't affect the truth value of sentences: For every $L$-sentence $\phi, w \in W_{M}$ and all variable-interpretations $s, s^{\prime}$ in $D_{M}$, we have that $V_{M}(\phi, w, s)=V_{M}\left(\phi, w, s^{\prime}\right)$. This means that in defining the three-place function $V_{M}$ pairing $L$-formulas, worlds and variable-interpretations with truth values, we have defined a binary function, call it $V S_{M}$, pairing each $L$-sentence 
and element of $W_{M}$ with a unique truth value, as required by the atomistic approach. For every $w \in W_{M}$, let's say that the sentential valuation generated by $M$ for $w$ is the function $v s_{M}^{w}$ defined as follows: For every $L$-sentence $\phi, v s_{M}^{w}(\phi)=V S_{M}(\phi, w)$.

Now we can define, like in nonmodal first-order logic, a relation of logical consequence for sentences of a modal first-order language, corresponding to the weakest normal modal system:

An $L$-sentence $\phi$ is a $K$-logical consequence of a set of $L$-sentences $\Gamma\left(\Gamma \vDash_{K} \phi\right)$ just in case for every $L$-model $M$ and every $w \in W_{M}$, if $V S_{M}(\gamma, w)=T$ for every $\gamma \in \Gamma$, then $\operatorname{VS}_{M}(\phi, w)=T$.

As in modal propositional logic, we can now define stronger relations of logical consequence by imposing conditions on the accessibility relation of the models that figure in the definition.

\subsection{The holistic approach}

The application of the holistic approach to modal first-order logic draws on the ideas that we developed to adapt the holistic template to nonmodal first-order logic and to modal propositional logic. As in nonmodal first-order logic, we start with a languagerelative definition of logical consequence and then define the relation that will do the job in terms of the language-relative relations on the onomastic expansions of the target language. As in modal propositional logic, we don't formulate necessary and sufficient conditions for a valuation to be admissible. We formulate necessary and sufficient conditions on sets of valuations. Admissible valuations are elements of sets that satisfy these conditions.

If $L$ is a modal first-order language, a set $V$ of q-Boolean $L$-valuations is $m q$ Boolean just in case it satisfies the following conditions:

( $\square$ ) For every $L$-sentence $\phi$ and every $v \in V, v(\square \phi)=T$ if for every $v^{\prime} \in V$ such that $v^{\prime}$ actualizes $v, v^{\prime}(\phi)=T$.

$(\doteq)$ For all individual constants $c, c^{\prime}$ of $L$ and all $v, v^{\prime} \in V, v\left(c \doteq c^{\prime}\right)=v^{\prime}\left(c \doteq c^{\prime}\right)$.

Condition $(\square)$ is the same as in modal propositional logic. Condition $(\doteq)$ reflects the fact that in the atomistic semantics that we are seeking to replicate, individual constants have the same denotation in every possible world. Notice, though, that $(\dot{=})$ is not needed to show that if $v\left(c \doteq c^{\prime}\right)=T$, then $v\left(\square c \doteq c^{\prime}\right)=T$. We have that $\square c \doteq c$ is $(\square c \doteq x)[c / x]$ and $\square c \doteq c^{\prime}$ is $(\square c \doteq x)\left[c^{\prime} / x\right]$. Hence, if $v\left(c \doteq c^{\prime}\right)=T$, then, since $\sigma(\square c \doteq c)=T$ for every $\sigma,(\doteq b)$ gives us $v\left(\square c \doteq c^{\prime}\right)=T$. However, $(\doteq)$ is needed to establish that if $v\left(\neg c \doteq c^{\prime}\right)=T$, then $v\left(\square \neg c \doteq c^{\prime}\right)=T$. If a set of q-Boolean valuations satisfying $(\square)$ contains valuations $v, v^{\prime}$ such that $v\left(c \doteq c^{\prime}\right)=$ $T, v^{\prime}\left(c \doteq c^{\prime}\right)=F$, the argument we've just given shows that $v^{\prime}$ cannot actualize $v$, but $v$ may well actualize $v^{\prime}$, so that $v^{\prime}\left(\neg c \doteq c^{\prime}\right)=T, v^{\prime}\left(\square \neg c \doteq c^{\prime}\right)=F .{ }^{9}$

We can now define a language-relative relation of logical consequence corresponding to the weakest normal modal system:

\footnotetext{
9 Instead of $(\doteq)$ we could require that for every valuation $v$ in an mq-Boolean set, if $v\left(\neg c \doteq c^{\prime}\right)=T$, then $v\left(\square \neg c \doteq c^{\prime}\right)=T$. However, having $(\doteq)$ will facilitate the proof of the equivalence of holistic and atomistic logical consequence.
} 
An $L$-sentence $\phi$ is an $L$ - $K$-logical consequence of a set of $L$-sentences $\Gamma\left(\Gamma \models_{K}^{L} \phi\right)$ just in case for every mq-Boolean set of $L$-valuations $V$ and every $v \in V$, if $v(\gamma)=T$ for every $\gamma \in \Gamma$, then $v(\phi)=T$.

For the same reasons given in connection with nonmodal first-order languages, this won't do as a definition of logical consequence for $L$. We overcome the difficulty in the same way:

An $L$-sentence $\phi$ is a $K$-logical consequence of a set of $L$-sentences $\Gamma\left(\Gamma \vDash_{K} \phi\right)$ just in case for every onomastic expansion $L^{\prime}$ of $L, \Gamma \vDash_{K}^{L^{\prime}} \phi$.

As we did with modal propositional logic, we can define relations of logical consequence corresponding to stronger modal systems, either by imposing restrictions on the actualization relation of the mq-Boolean sets in the definiens, or by imposing conditions on the $L$-valuations in an mq-Boolean set.

In Sect. 7.3, below, we show that this holistic definition of logical consequence for modal first-order languages is equivalent to the atomistic definition provided above. The claim can be expressed as follows:

Theorem 3 Let $\phi$ be a sentence of a modal first-order language $L$ and let $\Gamma$ be a set of L-sentences. The following are equivalent:

1. For every onomastic expansion $L^{\prime}$ of $L$, for every mq-Boolean set $V$ of $L^{\prime}$-valuations and every $v \in V$, if $v(\gamma)=T$ for every $\gamma \in \Gamma$, then $v(\phi)=T$.

2. For every $L$-model $M$ and every $w \in W_{M}$, if $V S_{M}(\gamma, w)=T$ for every $\gamma \in \Gamma$, then $\operatorname{VS}_{M}(\phi, w)=T$.

\section{Atomism or holism?}

The atomistic approach and the holistic approach correspond to two different conceptions of the source of logical properties and relations, such as logical consequence. It is widely accepted that language makes contact with reality at the level of its atomic constituents. Compound sentences obtain their semantic properties from their components, and ultimately from the atomic constituents that figure in them. The definitions of truth for formal languages provide us with the tools for investigating this phenomenon.

By using these same tools for explicating logical consequence, the atomistic approach presents the concept as arising from the mechanisms by which language comes to represent the world. A sentence $\phi$ follows from a set of sentences $\Gamma$, on this approach, when the possible ways in which $\phi$ and the elements of $\Gamma$ might represent the world don't include any in which $\phi$ comes out false and all the elements of $\Gamma$ come out true.

On the holistic approach, by contrast, logical properties and relations are not connected with the mechanisms through which language makes contact with the world.

In systems at least as strong as $B,(\doteq)$ is not needed to establish that if $v\left(\neg c \doteq c^{\prime}\right)=T$, then $v(\square \neg c \doteq$ $\left.c^{\prime}\right)=T$ either. In these systems, every valuation $v$ in an mq-Boolean set $V$ is such that if $v(\neg \phi)=T$, then $v(\square \neg \square \phi)=T$. Hence, if $v\left(\neg c \doteq c^{\prime}\right)=T$, then for every valuation $v^{\prime} \in V$ that actualizes $v$ we have that $v^{\prime}\left(\square c \doteq c^{\prime}\right)=F$. But we have seen that if $v^{\prime}\left(c \doteq c^{\prime}\right)=T$, then $v^{\prime}\left(\square c \doteq c^{\prime}\right)=T$. Therefore, for every $v^{\prime} \in V$ that actualizes $v$ we have that $v^{\prime}\left(c \doteq c^{\prime}\right)=F$, and $v\left(\square \neg c \doteq c^{\prime}\right)=T$. 
They arise instead from factors internal to language - from the fact that some combinations of truth values for sentences are incompatible with structural features of these sentences. Atomic components play no special role in the account, and the ways in which these make contact with the world play no role whatsoever.

This is not the place to adjudicate the contest between these conceptions of the source of logical properties and relations. My goal is to show that the second conception can produce workable definitions of logical consequence for standard formal languages. This result, established in the next section, will block what some may have seen as a powerful argument for the atomistic approach. The premise of this argument is the thought that what makes $\phi$ a logical consequence of $\Gamma$ is the fact that structural features of $\phi$ and the elements of $\Gamma$ make it impossible for $\phi$ to be false if all the elements of $\Gamma$ are true. ${ }^{10}$ This premise would render the atomistic approach unavoidable if the explanation of how the structures of propositions rule out certain truth-value combinations needed to invoke how their truth values are determined by the semantic values of their atomic components. The availability of the holistic approach shows that this isn't the case. We can explain how the structures of propositions rule out certain truth-value combinations without considering how their truth values are determined by the semantic values of their atomic components. Hence the holistic approach deprives the atomistic approach of this line of support.

Notice, in addition, that the resources deployed by the atomistic approach for explaining logical consequence go well beyond what is required for the task. I'm going to illustrate the point with the case of nonmodal first-order logic, although it applies in the same way to modal first-order logic. In order to define logical consequence for a first-order language $L$, all we need to do is specify which $L$-valuations are compatible with the intended interpretations of the logical constants. Hence the value of $L$-structures for the task of defining logical consequence is restricted to the fact that each $L$-structure singles out a unique $L$-valuation. But $L$-structures do much more than this, as can be seen by the fact that (infinitely) many different non-isomorphic $L$-structures single out the same $L$-valuation. This is a direct consequence of what is usually presented as the expressive limitations of first-order logic. Thus it follows from the Löwenheim-Skolem results that if $\mathfrak{A}$ is an $L$-structure with an infinite universe, then for every infinite cardinality $\kappa$ greater than or equal to the cardinality of $L$ there is an $L$-structure $\mathfrak{B}$ with a universe of cardinality $\kappa$ that singles out the same $L$-valuation as $\mathfrak{A} .{ }^{11}$ I.e., in the symbolism introduced in Sect. $4, v s_{\mathfrak{A}}=v s_{\mathfrak{B}} .{ }^{12}$ The ways in which $\mathfrak{A}$ might differ from $\mathfrak{B}$ are of the greatest importance in the study of the representational properties of $L$, but they are completely irrelevant for the task of defining logical consequence. ${ }^{13}$ From the point of view of parsimony, the holistic approach has a clear advantage over the atomistic approach.

\footnotetext{
10 This premise will be rejected by those who endorse proof-theoretic accounts of logical consequence.

11 See Zalabardo (2000, pp. 263-271).

12 Of course there are also cases in which $v s_{\mathfrak{A}}=v s_{\mathfrak{B}}$ and $\mathfrak{A}$ is not isomorphic to $\mathfrak{B}$ although their universes are of the same cardinality. This is the situation, for example, with non-standard models of first-order arithmetic. See Zalabardo (2000, pp. 272-282).

13 In Zalabardo (2018) I argue that we encounter a similar phenomenon in the atomistic treatment of modal propositional logic_different non-isomorphic models that generate the same m-Boolean set of $M P L$ valuations.
} 


\section{Equivalence results}

In this section I establish the results stated above, to the effect that the holistic definitions of logical consequence I have provided for modal propositional logic and nonmodal and modal first-order logic are equivalent to the atomistic alternatives.

\subsection{Modal propositional logic}

If $M$ is a modal model, the set of $M P L$-valuations generated by $M$ is the following set:

$$
\left\{v \text { : for some } w \in W_{M} \text {, for every } M P L-\text { sentence } \phi, V_{M}(\phi, w)=v(\phi)\right\}
$$

Lemma 1 A set of MPL-valuations is m-Boolean just in case it is generated by some modal model.

Proof Let $M$ be a modal model, and let $V$ be the set of $M P L$-valuations it generates. We need to show that $V$ is m-Boolean.

We can easily check that every element of $V$ satisfies the clauses of the definition of Boolean valuation. We need to show, in addition, that for every $v \in V$, if for every $v^{\prime} \in V$ that actualizes $v, v^{\prime}(\phi)=T$, then $v(\square \phi)=T$.

Let $v \in V$ and let $w$ be such that for every MPL-sentence $\phi, v(\phi)=V_{M}(\phi, w)$. Assume that for every $v^{\prime} \in V$ that actualizes $v, v^{\prime}(\phi)=T$. We need to show that $v(\square \phi)=T$. We argue as follows:

For every $v^{\prime} \in V$ that actualizes $v, v^{\prime}(\phi)=T$

$\Downarrow$ (since if $w R w^{\prime}$ and $v^{\prime}$ is such that for every $M P L$-sentence $\phi, v^{\prime}(\phi)=$ $V_{M}\left(\phi, w^{\prime}\right)$, then $v^{\prime}$ actualizes $\left.v\right)$

For every $w^{\prime} \in W_{M}$ such that $w R w^{\prime}, V_{M}\left(\phi, w^{\prime}\right)=T$

$\Downarrow\left(\right.$ definition of $\left.V_{M}\right)$

$V_{M}(\square \phi, w)=T$

$\Downarrow$

$v(\square \phi)=T$

Let $V$ now be an m-Boolean set of $M P L$-valuations. Let $M$ be the modal model defined as follows:

$-W_{M}=V$.

- For all $v, v^{\prime} \in W_{M}, v R_{M} v^{\prime}$ just in case $v^{\prime}$ actualizes $v$.

- For every atom $\alpha$ and every $v \in W_{M}, A_{M}(\alpha, v)=v(\alpha)$.

We show that $M$ is a modal model that generates $V$. For this we show by induction on $M P L$-sentences that for every $M P L$-sentence $\phi$ and every $v \in V, V_{M}(\phi, v)=v(\phi)$.

For the inductive clause for $\square$ we assume (IH) that for every $v^{\prime} \in V, V_{M}\left(\phi, v^{\prime}\right)=$ $v^{\prime}(\phi)$. We need to show that for every $v \in V, V_{M}(\square \phi, v)=v(\square \phi)$. We argue as follows:

$V_{M}(\square \phi, v)=T$

企 (definition of $V_{M}$ ) 
For every $v^{\prime} \in V$ such that $v R_{M} v^{\prime}, V_{M}\left(\phi, v^{\prime}\right)=T$

企 (IH)

For every $v^{\prime} \in V$ such that $v R_{M} v^{\prime}, v^{\prime}(\phi)=T$

企 (definition of $R_{M}$ )

For every $v^{\prime} \in V$ that actualizes $v, v^{\prime}(\phi)=T$

业 ( $\Downarrow$ since $V$ is m-Boolean; $\Uparrow$ by the definition of actualization)

$v(\square \phi)=T$

The equivalence of the atomistic and holistic definitions of K-logical consequence expresssed by Theorem 1 is a straightforward corollary of Lemma 1.

\subsection{First-order logic}

We show first that holistic logical consequence entails atomistic logical consequence. For this purpose we'll need to invoke some preliminary results.

Lemma 2 If $\mathfrak{A}$ is an L-structure, s a variable-interpretation in $\mathfrak{A}, x$ a variable and $c$ an individual constant of $L$, then for every $L$-formula $\phi$,

$$
v_{\mathfrak{A}}\left(\phi, s_{\left(x / c_{\mathfrak{A}}\right)}\right)=v_{\mathfrak{A}}((\phi)[c / x], s) .
$$

Proof By induction on $L$-formulas. See, e.g., Zalabardo (2000, pp. 155-157).

We show next that the sentential valuations generated by certain structures are qBoolean. If $L$ is a first-order language, $\mathfrak{A}$ is an $L$-structure, and $C$ is a set of individual constants not in $L$ of the same cardinality as the universe $A$ of $\mathfrak{A}$, let $L^{+}$be the onomastic expansion of $L$ that we obtain by adding the elements of $C$ to the set of individual constants of $L$. And let $\mathfrak{A}^{+}$be the $L^{+}$-structure that we get from $\mathfrak{A}$ by adding: for every $c \in C, c_{\mathfrak{A}^{+}}=f(c)$, for some one-to-one correspondence $f$ between $C$ and $A$.

Lemma 3 If $L$ is a first-order language and $\mathfrak{A}$ is an L-structure, then $v s_{\mathfrak{A}^{+}}$is a $q$ Boolean $L^{+}$-valuation.

Proof We need to show that $v s_{\mathfrak{A}^{+}}$satisfies the clauses of the definition of q-Boolean $L^{+}$-valuation. We provide the arguments for $(\forall)$ and $(\doteq b)$.

For $(\forall)$ we argue as follows:

$v s_{\mathfrak{A}^{+}}(\forall x \phi)=T$

企 (definition of $v s_{\mathfrak{A}^{+}}$)

$v_{\mathfrak{A}^{+}}(\forall x \phi, s)=T$, for any variable-interpretation $s$ in $\mathfrak{A}$

企 (definition of $v_{\mathfrak{A}^{+}}$)

For every $a \in A, v_{\mathfrak{A}^{+}}\left(\phi, s_{(x / a)}\right)=T$, for any variable-interpretation $s$ in $\mathfrak{A}$

业 (since every individual constant of $L^{+}$denotes an element of $A$ and every element of $A$ is denoted by some individual constant of $L^{+}$)

For every individual constant $c$ of $L^{+}, v_{\mathfrak{A}^{+}}\left(\phi, s_{\left(x / c_{\mathfrak{A}^{+}}\right)}\right)=T$, for any variable-interpretation $s$ in $\mathfrak{A}$

企 (Lemma 2) 
For every individual constant $c$ of $L^{+}, v_{\mathfrak{A}^{+}}((\phi)[c / x], s)=T$, for any variable-interpretation $s$ in $\mathfrak{A}$

$$
\text { 企 (definition of } v s_{\mathfrak{A}^{+}} \text {) }
$$

For every individual constant $c$ of $L^{+}, v s_{\mathfrak{A}^{+}}((\phi)[c / x])=T$

For $(\doteq b)$ we argue as follows:

$$
\begin{aligned}
v s_{\mathfrak{A}^{+}} & \left(c \doteq c^{\prime}\right)=T \\
& \Downarrow\left(\text { definition of } v s_{\mathfrak{A}^{+}}\right)
\end{aligned}
$$

$v_{\mathfrak{A}^{+}}\left(c \doteq c^{\prime}, s\right)=T$, for any variable-interpretation $s$ in $\mathfrak{A}$

$\Downarrow$ (definition of $v_{\mathfrak{A}^{+}}$)

$\operatorname{den}_{\mathfrak{A}^{+}}(c, s)=\operatorname{den}_{\mathfrak{A}^{+}}\left(c^{\prime}, s\right)$, for any variable-interpretation $s$ in $\mathfrak{A}$

$c_{\mathfrak{A}^{+}}=\underset{c^{\prime}}{c^{+}}$

$\Downarrow\left(\right.$ definition of $\left.\operatorname{den}_{\mathfrak{A}^{+}}\right)$

$v_{\mathfrak{A}^{+}}\left(\phi, s_{\left(x / c_{\mathfrak{A}^{+}}\right)}\right)=v_{\mathfrak{A}^{+}}\left(\phi, s_{\left(x / c_{\mathfrak{A}^{+}}^{\prime}\right)}\right)$, for any variable-interpretation $s$ in $\mathfrak{A}$ and every $L^{+}$-formula $\phi$ in which only $x$ is free

$\Downarrow($ Lemma 2)

$v_{\mathfrak{A}^{+}}((\phi)[c / x], s)=v_{\mathfrak{A}^{+}}\left((\phi)\left[c^{\prime} / x\right], s\right)$, for any variable-interpretation $s$ in $\mathfrak{A}$ and every $L^{+}$-formula $\phi$ in which only $x$ is free

$\Downarrow\left(\right.$ definition of $\left.v s_{\mathfrak{A}^{+}}\right)$

$v s_{\mathfrak{A}^{+}}((\phi)[c / x])=v s_{\mathfrak{A}^{+}}\left((\phi)\left[c^{\prime} / x\right]\right)$, for every $L^{+}$-formula $\phi$ in which only $x$ is free

Lemma 4 Let $L$ be a first-order language and let $L^{\prime}$ be an onomastic expansion of $L$. Let $\mathfrak{A}^{\prime}$ be an $L^{\prime}$-structure, and let $\mathfrak{A}$ be the L-structure that we obtain by removing from $\mathfrak{A}^{\prime}$ the interpretations of the new constants of of $L^{\prime}$.

1. For every L-term $t$ and every variable-interpretation $s$ in $\mathfrak{A}$,

$$
\operatorname{den}_{\mathfrak{A}}(t, s)=\operatorname{den}_{\mathfrak{A}^{\prime}}(t, s)
$$

2. For every $L$-formula $\phi$ and every variable-interpretation s in $\mathfrak{A}$,

$$
v_{\mathfrak{A}}(\phi, s)=v_{\mathfrak{A}^{\prime}}(\phi, s)
$$

Proof 2 by induction on $L$-formulas.

We are now in a position to show that holistic logical consequence entails atomistic logical consequence.

Theorem 4 Let $\phi$ be a sentence of a first-order language $L$ and let $\Gamma$ be a set of L-sentences. 1 entails 2:

1. For every onomastic expansion $L^{\prime}$ of $L$, for every $q$-Boolean $L^{\prime}$-valuation $v$, if $v(\gamma)=T$ for every $\gamma \in \Gamma$, then $v(\phi)=T$.

2. For every L-structure $\mathfrak{A}$, if $v s_{\mathfrak{A}}(\gamma)=T$ for every $\gamma \in \Gamma$, then $v s_{\mathfrak{A}}(\phi)=T$. 
Proof Assume 1, and let $\mathfrak{A}$ be an $L$-structure such that $v s_{\mathfrak{A}}(\gamma)=T$ for every $\gamma \in \Gamma$. We need to prove that $v s_{\mathfrak{A}}(\phi)=T$. We argue as follows:

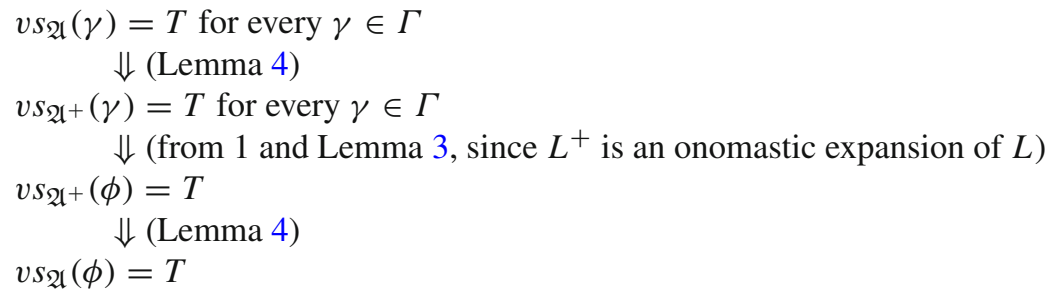

We turn now to showing that atomistic logical consequence entails holistic logical consequence.

Let $L$ be a first-order language with at least one individual constant, and let $v$ be a q-Boolean $L$-valuation. Let $E$ be the relation on the set of individual constants of $L$ defined as follows: For all individual constants $c_{1}, c_{2}, c_{1} E c_{2}$ if and only if $v\left(c_{1} \doteq\right.$ $\left.c_{2}\right)=T$. It follows directly from the following result that $E$ is an equivalence relation.

Lemma 5 If $L$ is a first-order language and $v$ a q-Boolean L-valuation, then, for all individual constants $c, d$, e the following hold:

1. $v(c \doteq c)=T$

2. $v(c \doteq d)=v(d \doteq c)$

3. If $v(c \doteq d)=v(d \doteq e)=T$, then $v(c \doteq e)=T$

Proof 1 follows directly from the definition of q-Boolean valuation.

For 2 we argue as follows:

$v(c \doteq d)=T$

$\Downarrow$ (since $v$ is q-Boolean)

For every $L$-formula $\phi$ in which only $x$ is free, $v((\phi)[c / x])=v((\phi)[d / x])$

$\Downarrow$ (since $x \doteq c$ is an $L$-formula in which only $x$ is free, $v((x \doteq c)[c / x])=T$ and $d \doteq c$ is $(x \doteq c)[d / x])$

$v(d \doteq c)=T$

For 3 we argue as follows:

$$
\begin{gathered}
v(c \doteq d)=v(d \doteq e)=T \\
\Downarrow(\text { since } v \text { is q-Boolean) }
\end{gathered}
$$

For every $L$-formula $\phi$ in which only $x$ is free,

$$
\begin{gathered}
v((\phi)[c / x]) \\
\Downarrow
\end{gathered}
$$

For every $L$-formula $\phi$ in which only $x$ is free, $v((\phi)[c / x])=v((\phi)[e / x])$

$\Downarrow($ since $c \doteq x$ is an $L$-formula in which only $x$ is free, $v((c \doteq x)[c / x])=T$ and $c \doteq e$ is $(c \doteq x)[e / x])$

$v(c \doteq e)=T$

Let $[c]_{E}$ denote the equivalence class generated by $c$ with $E$. Now, if $v$ is a qBoolean $L$-valuation, the Henkin structure generated by $v$ is the $L$-structure $\mathfrak{A}_{v}$ defined as follows: 
- The universe $A_{v}$ of $\mathfrak{A}_{v}$ is the set of equivalence classes generated by $E$.

- For every individual constant $c$ of $L, c_{\mathfrak{A}_{v}}=[c]_{E}$.

- For every $n$-place predicate $P$ of $L,\left\langle\left[c_{1}\right]_{E}, \ldots,\left[c_{n}\right]_{E}\right\rangle \in P_{\mathfrak{A}_{v}}$ if and only if $v\left(P c_{1} \ldots c_{n}\right)=T$.

Lemma 6 If $L$ is a first-order language with at least one individual constant and $v$ a $q$-Boolean $L$-valuation, then for every $L$-sentence $\phi$ and every variable-interpretation $s$ in $\mathfrak{A}_{v}, v(\phi)=v_{\mathfrak{A}_{v}}(\phi, s)$.

Proof We define the rank of an $L$-formula $\phi, r(\phi)$, by the following recursion:

- For every atomic $L$-formula $\phi, r(\phi)=1$.

- For every $L$-formula $\phi, r(\neg \phi)=r(\forall x \phi)=r(\phi)+1$.

- For all $L$-formulas $\phi, \psi, r(\phi \wedge \psi)=\operatorname{Max}(r(\phi), r(\psi))+1$.

We can establish the result by strong induction on the rank of a formula in the following form: for every $L$-formula $\phi$ and every variable-interpretation $s$ in $\mathfrak{A}_{v}$, if $\phi$ is an $L$ sentence, then $v(\phi)=v_{\mathfrak{A}_{v}}(\phi, s)$. For the inductive step, we assume (IH) that the result holds for every formula of rank no greater than $n$. We show that it holds for formulas of rank $n+1$. We provide the argument for $\forall$.

Let $\forall x \phi$ be an $L$-sentence of rank $n+1$. By the definition of rank, for every individual constant $c$ of $L,(\phi)[c / x]$ is an $L$-sentence of rank $n$ and by IH the result holds for $(\phi)[c / x]$. We argue as follows:

$$
\begin{aligned}
v_{\mathfrak{A}_{v}} & \forall x \phi, s)=T \\
& \text { 文 (definition of } \left.v_{\mathfrak{A}}\right)
\end{aligned}
$$

For every $a \in A_{v}, v_{\mathfrak{A}_{v}}\left(\phi, s_{(x / a)}\right)=T$

弯 (since for every individual constant $c, c_{\mathfrak{A}_{v}} \in A_{v}$, and every $a \in A_{v}$ is $c_{\mathfrak{A}_{v}}$ for some individual constant $c$ )

For every individual constant $c$ of $L, v_{\mathfrak{A}_{v}}\left(\phi, s_{\left(x / c_{\mathfrak{A}_{v}}\right)}\right)=T$

企 (Lemma 2)

For every individual constant $c$ of $L, v_{\mathfrak{A}_{v}}((\phi)[c / x], s)=T$

企 $(\mathrm{IH})$

For every individual constant $c$ of $L, v((\phi)[c / x])=T$

企 (since $v$ is q-Boolean)

$v(\forall x \phi)=T$

We can now prove that atomistic logical consequence entails holistic logical consequence.

Theorem 5 Let $\phi$ be a sentence of a first-order language $L$ and let $\Gamma$ be a set of sentences of L. 2 entails 1 :

1. For every onomastic expansion $L^{\prime}$ of $L$, for every $q$-Boolean $L^{\prime}$-valuation $v$, if $v(\gamma)=T$ for every $\gamma \in \Gamma$, then $v(\phi)=T$.

2. For every $L$-structure $\mathfrak{A}$, if $v s_{\mathfrak{A}}(\gamma)=T$ for every $\gamma \in \Gamma$, then $v s_{\mathfrak{A}}(\phi)=T$.

Proof Assume 2. Let $L^{\prime}$ be an onomastic expansion of $L$, let $v$ be a q-Boolean $L^{\prime}$ valuation such that $v(\gamma)=T$ for every $\gamma \in \Gamma$. We need to prove that $v(\phi)=T$. Let $\mathfrak{A}_{v}^{L}$ be the restriction to $L$ of the Henkin structure generated by $v, \mathfrak{A}_{v}$. 
We argue as follows:

$v(\gamma)=T$ for every $\gamma \in \Gamma$

$\Downarrow$ (Lemma 6)

$v_{\mathfrak{A}_{v}}(\gamma, s)=T$ for every $\gamma \in \Gamma$ and every variable interpretation $s$ in $\mathfrak{A}_{v}$ $\Downarrow$ (Lemma 4)

$v_{\mathfrak{A}_{v}^{L}}(\gamma, s)=T$ for every $\gamma \in \Gamma$ and every variable interpretation $s$ in $\mathfrak{A}_{v}^{L}$ $\Downarrow$ (from 2)

$v_{\mathfrak{A}_{v}^{L}}(\phi, s)=T$ for every variable interpretation $s$ in $\mathfrak{A}_{v}^{L}$

$\Downarrow$ (Lemma 4)

$v_{\mathfrak{A}_{v}}(\phi, s)=T$ for every variable interpretation $s$ in $\mathfrak{A}_{v}$ $\Downarrow($ Lemma 6)

$v(\phi)=T$

We have now attained our goal for the present section. It follows from Theorems 4 and 5 that atomistic logical consequence and holistic logical consequence are one and the same relation, as expressed by Theorem 2 .

\subsection{Modal first-order logic}

Our first goal is to show that holistic logical consequence entails atomistic logical consequence. We proceed in the same way as with nonmodal first-order logic.

Lemma 7 Let $L$ be a modal first-order language. If $M$ is an L-model , $w \in W_{M}$, $s$ a variable-interpretation in $M$ and $c$ an individual constant of $L$, then for every $L$-formula $\phi$,

$$
V_{M}\left(\phi, w, s_{\left(x / c_{M}\right)}\right)=V_{M}((\phi)[c / x], w, s) .
$$

Proof By induction on $L$-formulas. The base and the inductive clauses for $\neg, \wedge$ and $\forall$ are handled in the same way as in the proof of Lemma 2. We provide the clause for

Let $\phi$ be an $L$-formula. Assume (IH) that for every $w \in W_{M}$ and every variableinterpretation $s$ in $M, V_{M}\left(\phi, w, s_{\left(x / c_{M}\right)}\right)=V_{M}((\phi)[c / x], w, s)$. We need to show that for every $w \in W_{M}$ and every variable-interpretation $s$ in $M, V_{M}\left(\square \phi, w, s_{\left(x / c_{M}\right)}\right)=$ $V_{M}((\square \phi)[c / x], w, s)$. We argue as follows:

$V_{M}\left(\square \phi, w, s_{\left(x / c_{M}\right)}\right)=T$

企 (definition of $V_{M}$ )

For every $w^{\prime} \in W_{M}$ such that $w R_{M} w^{\prime}, V_{M}\left(\phi, w^{\prime}, s_{\left(x / c_{M}\right)}\right)=T$

企 $(\mathrm{IH})$

For every $w^{\prime} \in W_{M}$ such that $w R_{M} w^{\prime}, V_{M}\left((\phi)[c / x], w^{\prime}, s\right)=T$

立 (definition of $V_{M}$ )

$V_{M}(\square(\phi)[c / x], w, s)$

主 (definition of substitution)

$V_{M}((\square \phi)[c / x], w, s)$ 
We show next that the sets of sentential valuations generated by certain structures are mq-Boolean. If $L$ is a modal first-order language, $M$ is an $L$-structure, and $C$ is a set of individual constants not in $L$ of the same cardinality as the universe $D_{M}$ of $M$, let $L^{+}$be the the onomastic expansion of $L$ that we obtain by adding the elements of $C$ to the set of individual constants of $L$. And let $M^{+}$be the $L^{+}$-structure that we get from $M$ by adding, for every $c \in C, c_{M^{+}}=f(c)$, for some one-to-one correspondence $f$ between $C$ and $D_{M}$.

Lemma 8 If $L$ is a modal first-order language and $M$ is an L-structure, then $\left\{v s_{M^{+}}^{w}\right.$ : $w \in W_{M^{+}}$is a mq-Boolean set of $L^{+}$-valuations.

Proof We first need to show that, for every $w \in W_{M^{+}}, v s_{M^{+}}^{w}$ is q-Boolean. Let $w \in$ $W_{M^{+}}$. We provide the arguments for clauses $(\forall)$ and $(\doteq b)$ of the definition.

For $(\forall)$ we argue as follows:

$v s_{M^{+}}^{w}(\forall x \phi)=T$

业 (definition of $v s_{M^{+}}^{w}$ )

$V S_{M^{+}}(\forall x \phi, w)=T$

步 (definition of $V S_{M^{+}}$)

$V_{M^{+}}(\forall x \phi, w, s)=T$, for any variable-interpretation $s$ in $M^{+}$

企 (definition of $V_{M^{+}}$)

For every $a \in D_{M^{+}}, V_{M^{+}}\left(\phi, w, s_{(x / a)}\right)=T$, for any variable-interpretation $s$ in $M^{+}$ 企 (since every individual constant of $L^{+}$denotes an element of $D_{M^{+}}$and every element of $D_{M^{+}}$is denoted by some individual constant of $L^{+}$)

For every individual constant $c$ of $L^{+}, V_{M^{+}}\left(\phi, w, s_{\left(x / c_{M^{+}}\right)}\right)=T$, for any variableinterpretation $s$ in $\mathrm{M}^{+}$

立 (Lemma 7)

For every individual constant $c$ of $L^{+}, V_{M^{+}}((\phi)[c / x], w, s)=T$, for any variableinterpretation $s$ in $\mathrm{M}^{+}$

企 (definition of $V S_{M^{+}}$)

For every individual constant $c$ of $L^{+}, V S_{M^{+}}((\phi)[c / x], w)=T$

企 (definition of $v s_{M^{+}}^{w}$ )

For every individual constant $c$ of $L^{+}, v s_{M^{+}}^{w}((\phi)[c / x])=T$

For $(\doteq b)$ we argue as follows:

$v s_{M^{+}}^{w}\left(c \doteq c^{\prime}\right)=T$

$\Downarrow\left(\right.$ definition of $\left.v s_{M^{+}}^{w}\right)$

$V S_{M^{+}}\left(c \doteq c^{\prime}, w\right)=T$

$\Downarrow\left(\right.$ definition of $\left.V S_{M^{+}}\right)$

$V_{M^{+}}\left(c \doteq c^{\prime}, w, s\right)=T$, for any variable-interpretation $s$ in $M^{+}$

$\Downarrow\left(\right.$ definition of $\left.V_{M^{+}}\right)$

$\operatorname{den}_{M^{+}}(c, w, s)=\operatorname{den}_{M^{+}}\left(c^{\prime}, w, s\right)$, for any variable-interpretation $s$ in $M^{+}$

$\Downarrow$ (definition of $\operatorname{den}_{M^{+}}$)

$c_{M^{+}}=c_{\Downarrow}^{\prime} M^{+}$

$V_{M^{+}}\left(\phi, w, s_{\left(x / c_{M^{+}}\right)}\right)=V_{M^{+}}\left(\phi, w, s_{\left(x / c_{M^{+}}^{\prime}\right)}\right)$, for any variable-interpretation $s$ in $M^{+}$ and every $L^{+}$-formula $\phi$ in which only $x$ is free 
$\Downarrow($ Lemma 7)

$V_{M^{+}}((\phi)[c / x], w, s)=V_{M^{+}}\left((\phi)\left[c^{\prime} / x\right], w, s\right)$, for any variable-interpretation $s$ in $M^{+}$and every $L^{+}$-formula $\phi$ in which only $x$ is free

$\Downarrow$ (definition of $V S_{M^{+}}$)

$V S_{M^{+}}((\phi)[c / x], w)=V S_{M^{+}}\left((\phi)\left[c^{\prime} / x\right], w\right)$, for every $L^{+}$-formula $\phi$ in which only $x$ is free

$\Downarrow\left(\right.$ definition of $\left.v s_{M^{+}}^{w}\right)$

$v s_{M^{+}}^{w}((\phi)[c / x])=v s_{M^{+}}^{w}\left((\phi)\left[c^{\prime} / x\right]\right)$, for every $L^{+}$-formula $\phi$ in which only $x$ is free

Now we need to show that $\left\{v s_{M^{+}}^{w}: w \in W_{M^{+}}\right\}$satisfies the conditions for a set of q-Boolean $L$-valuations to be mq-Boolean.

( $\square$ ) Let $\phi$ be an $L$-sentence and let $w \in W_{M^{+}}$. We argue as follows:

For every $w^{\prime} \in W_{M^{+}}$such that $v s_{M^{+}}^{w^{\prime}}$ actualizes $v s_{M^{+}}^{w}, v s_{M^{+}}^{w^{\prime}}(\phi)=T$

$\Downarrow\left(\right.$ definition of $\left.v s_{M^{+}}^{w}\right)$

For every $w^{\prime} \in W_{M^{+}}$such that $v s_{M^{+}}^{w^{\prime}}$ actualizes $v s_{M^{+}}^{w}, V S_{M^{+}}\left(\phi, w^{\prime}\right)=T$

$\Downarrow\left(\right.$ definition of $\left.V S_{M^{+}}\right)$

For every $w^{\prime} \in W_{M^{+}}$such that $v s_{M^{+}}^{w^{\prime}}$ actualizes $v s_{M^{+}}^{w}, V_{M^{+}}\left(\phi, w^{\prime}, s\right)=T$ for any variable-interpretation $s$ in $M^{+}$

$\Downarrow$ (since accessibility entails actualization)

For every $w^{\prime} \in W_{M^{+}}$such that $w R_{M^{+}} w^{\prime}, V_{M^{+}}\left(\phi, w^{\prime}, s\right)=T$ for any variableinterpretation $s$ in $M^{+}$

$\Downarrow$ (definition of $V_{M^{+}}$)

$V_{M^{+}}(\square \phi, w, s)=T$ for any variable-interpretation $s$ in $M^{+}$

$\Downarrow\left(\right.$ definition of $\left.V S_{M^{+}}\right)$

$V S_{M^{+}}(\square \phi, w)=T$

$\Downarrow\left(\right.$ definition of $\left.v s_{M^{+}}^{w}\right)$

$v s_{M^{+}}^{w}(\square \phi)=T$

$(\doteq)$ Let $c, c^{\prime}$ be individual constants of $L^{+}$and let $w, w^{\prime} \in W_{M^{+}}$. We argue as follows:

$$
\begin{aligned}
v s_{M^{+}}^{w} & \left(c \doteq c^{\prime}\right)=T \\
& \text { 步 }\left(\text { definition of } v s_{M^{+}}^{w}\right) \\
V S_{M^{+}} & \left(c \doteq c^{\prime}, w\right)=T \\
& \text { 企 (definition of } \left.V S_{M^{+}}\right)
\end{aligned}
$$

$V_{M^{+}}\left(c \doteq c^{\prime}, w, s\right)=T$, for any variable-interpretation $s$ in $M^{+}$

企 (definition of $V_{M^{+}}$)

$\operatorname{den}_{M^{+}}(c, w, s)=\operatorname{den}_{M^{+}}\left(c^{\prime}, w, s\right)$, for any variable-interpretation $s$ in $M^{+}$

企 (definition of $\operatorname{den}_{M^{+}}$)

$c_{M^{+}}=c_{M^{+}}^{\prime}$

步 (definition of $\operatorname{den}_{M^{+}}$)

$\operatorname{den}_{M^{+}}\left(c, w^{\prime}, s\right)=\operatorname{den}_{M^{+}}\left(c^{\prime}, w^{\prime}, s\right)$, for any variable-interpretation $s$ in $M^{+}$

企 (definition of $V_{M^{+}}$)

$V_{M^{+}}\left(c \doteq c^{\prime}, w^{\prime}, s\right)=T$, for any variable-interpretation $s$ in $M^{+}$

企 (definition of $V S_{M^{+}}$)

$V S_{M^{+}}\left(c \doteq c^{\prime}, w^{\prime}\right)=T$

企 (definition of $v s_{M^{+}}^{w^{\prime}}$ ) 
$v s_{M^{+}}^{w^{\prime}}\left(c \doteq c^{\prime}\right)=T$

Lemma 9 Let $L$ be a modal first-order language and let $L^{\prime}$ be an onomastic expansion of L. Let $M^{\prime}$ be an $L^{\prime}$ structure, and let $M$ be the $L$-structure that we obtain by removing from $M^{\prime}$ the interpretations of the symbols of $L^{\prime}$ not in $L$.

1. For every L-term $t$, every $w \in W_{M}$ and every variable-interpretation $s$ in $M$, $\operatorname{den}_{M}(t, w, s)=\operatorname{den}_{M^{\prime}}(t, w, s)$.

2. For every $L$-formula $\phi$, every $w \in W_{M}$ and every variable-interpretation $s$ in $M$, $v_{M}(\phi, w, s)=v_{M^{\prime}}(\phi, w, s)$.

Proof 2 by induction on $L$-formulas.

We can now establish that holistic $K$-logical consequence entails atomistic $K$ logical consequence.

Theorem 6 Let $\phi$ be a sentence of a modal first-order language $L$ and let $\Gamma$ be a set of sentences of L. 1 entails 2 :

1. For every onomastic expansion $L^{\prime}$ of $L$, for every mq-Boolean set $V$ of $L^{\prime}$ valuations and every $v \in V$, if $v(\gamma)=T$ for every $\gamma \in \Gamma$, then $v(\phi)=T$.

2. For every L-model $M$ and every $w \in W_{M}$, if $V S_{M}(\gamma, w)=T$ for every $\gamma \in \Gamma$, then $\operatorname{VS}_{M}(\phi, w)=T$.

Proof Assume 1, and let $M$ be an $L$-model and $w \in W_{M}$ such that $V S_{M}(\gamma, w)=T$ for every $\gamma \in \Gamma$. We need to prove that $\operatorname{VS}_{M}(\phi, w)=T$. We argue as follows:

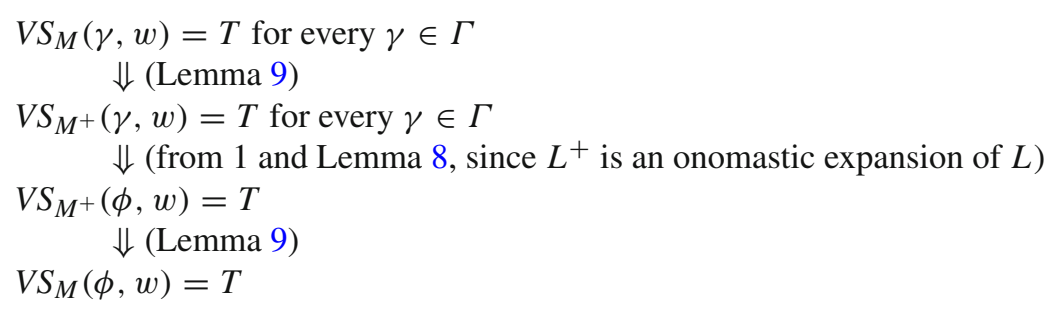

We turn now to our final task of establishing that atomistic $K$-logical consequence entails holistic $K$-logical consequence.

Let $L$ be a modal first-order language with at least one individual constant, and let $V$ be an mq-Boolean set of $L$-valuations. Let $E$ be the relation on the set of individual constants of $L$ defined as follows: For all individual constants $c_{1}, c_{2}, c_{1} E c_{2}$ if and only if $v\left(c_{1} \doteq c_{2}\right)=T$, for any $v$ in $V$. We can easily prove, as we did for nonmodal first-order logic, that $E$ is an equivalence relation. Let $[c]_{E}$ denote the equivalence class generated by $c$ with $E$.

Now, if $V$ is an mq-Boolean set of $L$-valuations, the Henkin model generated by $V$ is the $L$-model $M_{V}$ defined as follows:

$-W_{M_{V}}=V$.

- $D_{M_{V}}$ is the set of equivalence classes generated by $E$.

- $R_{M_{V}}$ is the actualization relation on $V$.

- For every individual constant $c$ of $L, c_{M_{V}}=[c]_{E}$. 
- For every $n$-place predicate $P$ of $L$ and every $v \in V,\left\langle\left[c_{1}\right]_{E}, \ldots,\left[c_{n}\right]_{E}\right\rangle \in P_{M_{V}}^{v}$ if and only if $v\left(P c_{1} \ldots c_{n}\right)=T$.

Lemma 10 If $L$ is a modal first-order language with at least one individual constant and $V$ is an $m q$-Boolean set of $L$-valuations, then for every $L$-sentence $\phi$ and every $v \in V, v(\phi)=V_{M_{V}}(\phi, v, s)$, for any variable-interpretation s in $M_{V}$.

Proof By strong induction on the rank of a formula (see proof of Lemma 6; add: $r(\square \phi)=r(\phi)+1)$, in the following form: for every $L$-formula $\phi$, every $v \in V$ and every variable-interpretation $s$ in $M_{V}$, if $\phi$ is a sentence, then $v(\phi)=v_{M_{V}}(\phi, v, s)$. The argument is the same as in the proof of Lemma 6 . We provide the inductive clauses for $\forall$ and $\square$.

Let $\forall x \phi$ be an $L$-sentence of rank $n+1$. By the definition of rank, for every individual constant $c,(\phi)[c / x]$ is an $L$-sentence of rank $n$ and by IH the result holds for $(\phi)[c / x]$. We argue as follows:

$$
\begin{aligned}
V_{M_{V}}( & \forall x \phi, v, s)=T \\
& \text { 步 (definition of } \left.V_{M}\right)
\end{aligned}
$$

For every $a \in D_{M_{V}}, V_{M_{V}}\left(\phi, v, s_{(x / a)}\right)=T$

企 (since every individual constant has an element of the universe as its denotation and every element of the universe is the denotation of some individual constant)

For every individual constant $c$ of $L, V_{M_{V}}\left(\phi, v, s_{\left(x / c_{M_{V}}\right)}\right)=T$

企 (Lemma 7)

For every individual constant $c$ of $L, V_{M_{V}}((\phi)[c / x], v, s)=T$

企 $(\mathrm{IH})$

For every individual constant $c$ of $L, v((\phi)[c / x])=T$

业 (since $v$ is q-Boolean)

$v(\forall x \phi)=T$

Let $\square \phi$ be an $L$-sentence of rank $n+1$. By the definition of rank, $\phi$ is an $L$ sentence of rank $n$ and by IH the result holds for $\phi$ : for every $v \in V$ and every variable-interpretation $s$ in $M_{V}, v(\phi)=V_{M_{V}}(\phi, v, s)$. We argue as follows:

$$
\begin{aligned}
& V_{M_{V}}(\square \phi, v, s)=T \\
& \text { 虫 (definition of } \left.V_{M}\right)
\end{aligned}
$$

For every $v^{\prime} \in V$ such that $v R_{M_{V}} v^{\prime}, V_{M_{V}}\left(\phi, v^{\prime}, s\right)=T$

企 (definition of $R_{M_{V}}$ )

For every $v^{\prime} \in V$ such that $v^{\prime}$ actualizes $v, V_{M_{V}}\left(\phi, v^{\prime}, s\right)=T$

$$
\text { 步 (IH) }
$$

For every $v^{\prime} \in V$ such that $v^{\prime}$ actualizes $v, v^{\prime}(\phi)=T$

企 (since $V$ is mq-Boolean)

$v(\square \phi)=T$

We can now establish that atomistic $K$-logical consequence entails holistic $K$-logical consequence.

Theorem 7 Let $\phi$ be a sentence of a modal first-order language $L$ and let $\Gamma$ be a set of sentences of L. 2 entails 1: 
1. For every onomastic expansion $L^{\prime}$ of $L$, for every $m q$-Boolean set $V$ of $L^{\prime}$-valuations and every $v \in V$, if $v(\gamma)=T$ for every $\gamma \in \Gamma$, then $v(\phi)=T$.

2. For every L-model $M$ and every $w \in W_{M}$, if $V S_{M}(\gamma, w)=T$ for every $\gamma \in \Gamma$, then $V S_{M}(\phi, w)=T$.

Proof Assume 2. Let $L^{\prime}$ be an onomastic expansion of $L$, let $V$ be an mq-Boolean set of $L^{\prime}$-valuations, and let $v$ be an $L^{\prime}$-valuation in $V$ such that $v(\gamma)=T$ for every $\gamma \in \Gamma$. We need to prove that $v(\phi)=T$. Let $M_{V}^{L}$ be the restriction to $L$ of $M_{V}$, the Henkin model generated by $V$.

We argue as follows:

$$
\begin{aligned}
& v(\gamma)=T \text { for every } \gamma \in \Gamma \\
& \Downarrow \text { (Lemma 10) } \\
& V_{M_{V}}(\gamma, v, s)=T \text { for every } \gamma \in \Gamma \text {, for any variable-interpretation } s \text { in } M_{V} \\
& \Downarrow\left(\text { definition of } V S_{M_{V}}\right) \\
& V S_{M_{V}}(\gamma, v)=T \text { for every } \gamma \in \Gamma \\
& \Downarrow \text { (Lemma 9) } \\
& V S_{M_{V}^{L}}(\gamma, v)=T \text { for every } \gamma \in \Gamma \\
& \Downarrow \text { (from 2) } \\
& V S_{M_{V}^{L}}(\phi, v)=T \\
& \Downarrow \text { (Lemma 9) } \\
& V S_{M_{V}}(\phi, v)=T \\
& \Downarrow\left(\text { definition of } V S_{M_{V}}\right) \\
& V_{M_{V}}(\phi, v, s)=T \text {, for any variable-interpretation } s \text { in } M_{V} \\
& \Downarrow(\text { Lemma 10) } \\
& v(\phi)=T
\end{aligned}
$$

It follows from Theorems 6 and 7 that the holistic definition of logical consequence for modal first-order logic is equivalent to the atomistic definition, as expressed by Theorem 3.

\section{Conclusion}

I have shown that the holistic approach can be successfully applied to the task of defining logical consequence in propositional and first-order logic, modal as well as nonmodal. I have shown that the resulting definitions are equivalent to the standard definitions based on the atomistic template. One salient feature of the holistic definitions I've provided is that they make no use of the technical apparatus of first-order, modal and first-order modal models, employed by the atomistic approach for this purpose. This technical apparatus is a fascinating subject of study in its own right and nothing I've said detracts from the interest of this study. What does follow from the results I've presented is that modal, first-order and modal first-order models are not required for defining logical consequence. In general, we can maintain that logical properties and relations arise from the fact that some combinations of truth values are incompatible with formal features of sentences, while rejecting the link between logical properties and relations and the mechanisms by which sentences come to represent the world. 
Acknowledgements For their comments on this material, I'm grateful to Kit Fine and Antti Hautamäki, to my colleagues Lavinia Picollo and Daniel Rothschild, and to two anonymous referees for this journal.

Open Access This article is distributed under the terms of the Creative Commons Attribution 4.0 International License (http://creativecommons.org/licenses/by/4.0/), which permits unrestricted use, distribution, and reproduction in any medium, provided you give appropriate credit to the original author(s) and the source, provide a link to the Creative Commons license, and indicate if changes were made.

\section{References}

Bonevac, D. (1984). Systems of substitutional semantics. Philosophy of Science, 51, 631-656.

Dunn, J. M., \& Belnap, N. D. (1968). The substitution interpretation of the quantifiers. Nous, 2, 177-185.

Garson, J. W. (1984). Quantification in modal logic. In D. Gabbay \& F. Guenthner (Eds.), Handbook of philosophical logic. Volume II: Extensions of classical logic (pp. 249-307). Dordrecht: Reidel.

Hintikka, J. (1955). Form and content in quantification theory. Acta Philosophica Fennica, 8, 7-55.

Kripke, S. (1963). Semantical analysis of modal logic I: Normal modal propositional calculi. Zeitschrift für mathematische Logik und Grundlagen der Mathematik, 9, 67-96.

Leblanc, H. (1976). Truth-value semantics. Amsterdam: North-Holland.

Leblanc, H. (1983). Alternatives to standard first-order semantics. In D. Gabbay \& F. Guenthner (Eds.), Handbook of philosophical logic. Volume I: Elements of classical logic (pp. 189-274). Dordrecht: Springer.

Linsky, B., \& Zalta, E. N. (1994). In defense of the simplest quantified modal logic. In J. Tomberlin (Ed.), Philosophical perspectives 8: Logic and language (pp. 431-458). Atascadero: Ridgeview Press.

Smullyan, R. M. (1968). First-order logic. New York: Springer.

Zalabardo, J. L. (2000). Introduction to the theory of logic. Boulder: Westview Press.

Zalabardo, J. L. (2018). Actualism and modal semantics. Acta Analytica, 33, 35-49.

Publisher's Note Springer Nature remains neutral with regard to jurisdictional claims in published maps and institutional affiliations. 